\title{
Medical Condition or Procedure Affects Implantation Site
}

National Cancer Institute

\section{Source}

National Cancer Institute. Medical Condition or Procedure Affects Implantation Site. NCI

Thesaurus. Code C99982.

The medical condition or the procedure affects the device implantation site. (ACC) 